DOI: $10.17516 / 1998-2836-0191$

УДК 541.138:547.1.13:546.719:546.92

\title{
Phenylvinylidene RePt Complexes Containing Diphenylphosphine Ligands at the Platinum Atom: \\ Synthesis, IR, NMR Spectroscopic \\ and Electrochemical Properties
}

\author{
Oleg S. Chudin, Victor V. Verpekin*, \\ Galina V. Burmakina, Dmitry V. Zimonin, \\ Alexander A. Kondrasenko and Anatoly I. Rubaylo \\ Institute of Chemistry and Chemical Technology SB RAS \\ FRC "Krasnoyarsk Science Center SB RAS" \\ Krasnoyarsk, Russin Federation
}

Received 23.03.2020, received in revised form 12.04.2020, accepted 18.08.2020

Abstract. The $\mu$-phenylvinylidene complexes $\mathrm{Cp}(\mathrm{CO})_{2} \operatorname{RePt}(\mu-\mathrm{C}=\mathrm{CHPh})\left(\mathrm{PPh}_{2} \mathrm{H}\right)(\mathrm{L})\left[\mathrm{L}=\mathrm{PPh}_{3}(1)\right.$, $\left.\mathrm{P}\left(\mathrm{OPr}^{\mathrm{i}}\right)_{3}(2)\right]$ were synthesized for the first time. Their IR, NMR spectroscopic and redox properties were studied. Based on IR, NMR spectroscopic data their molecular structures were proposed. The pathways of redox-reactions of the complexes were determined.

Keywords: electrochemistry, redox properties, binuclear complexes, phenylvinylidene, rhenium, platinum, carbonyl, diphenylphosphine.

Citation: Chudin O.S., Verpekin V.V., Burmakina G.V., Zimonin D.V., Kondrasenko A.A., Rubaylo A.I. Phenylvinylidene RePt complexes containing diphenylphosphine ligands at the platinum atom: synthesis, IR, NMR spectroscopic and electrochemical properties, J. Sib. Fed. Univ. Chem., 2020, 13(3), 385-394. DOI: 10.17516/1998-2836-0191

(c) Siberian Federal University. All rights reserved

This work is licensed under a Creative Commons Attribution-NonCommercial 4.0 International License (CC BY-NC 4.0).

* Corresponding author E-mail address: vvv@icct.ru,vvv@sany-ok.ru 


\title{
Фенилвинилиденовые RePt-комплексы \\ с дифенилфосфиновыми лигандами при атоме платины: \\ синтез, ИК, ЯМР-спектроскопические \\ и электрохимические свойства
}

\author{
О.С. Чудин, В.В. Верпекин, Г.В. Бурмакина, \\ Д.В. Зимонин, А.А. Кондрасенко, А.И. Рубайло \\ Институт химии и химической технологии \\ ФИЦ «Красноярский научный изентр СО РАН» \\ Российская Федерация, Красноярск
}

Аннотащия. Синтезированы новые $\mu$-фенилвинилиденовые комплексы $\mathrm{Cp}(\mathrm{CO})_{2} \operatorname{RePt}(\mu-\mathrm{C}=\mathrm{CHPh})$ $\left(\mathrm{PPh}_{2} \mathrm{H}\right)(\mathrm{L})\left[\mathrm{L}=\mathrm{PPh}_{3}(1), \mathrm{P}\left(\mathrm{OPr}^{\mathrm{i}}\right)_{3}\right.$ (2)]. Методами ИК, ЯМР-спектроскопии и электрохимии изучены их физико-химические свойства. На основании полученных данных предложено строение комплексов, установлена схема их редокс-превращений.

Ключевые слова: электрохимия, редокс-свойства, биядерные комплексы, фенилвинилиден, рений, платина, карбонил, дифенилфосфин.

Цитирование: Чудин, О.С. Фенилвинилиденовые RePt-комплексы с дифенилфосфиновыми лигандами при атоме платины: синтез, ИК, ЯМР-спектроскопические и электрохимические свойства / О.С. Чудин, В.В. Верпекин, Г.В. Бурмакина, Д.В. Зимонин, А.А. Кондрасенко, А.И. Рубайло // Журн. Сиб. федер. ун-та. Химия, 2020. 13(3). С. 385394. DOI: $10.17516 / 1998-2836-0191$

\section{Введение}

Внимание исследователей к гетерометаллическим комплексам и кластерам переходных металлов с мостиковыми ненасыщенными углеводородными лигандами обусловлено возможностями их применения в различных областях современной химии [1-6]. Наличие различных атомов металлов в одной молекуле делает гетерометаллические соединения более эффективными и интересными катализаторами, чем комплексы на основе одного металла $[2,3]$. Кроме того, благодаря строго определенному соотношению атомов металлов в гетерометаллических соединениях они могут выступать в качестве прекурсоров полиметаллических материалов с точным распределением металлов [4]. Наиболее известные методы синтеза таких соединений основаны на взаимодействии непредельных углеводородов (алкены, алкины) с уже сформированными гетерометаллическими системами $[5,6]$, а также на последовательном увеличении нуклеарности их металлоостова в результате присоединения координационно-ненасыщенных металлофрагментов по связям $\mathrm{M}=\mathrm{C}$ и $\mathrm{C}=\mathrm{C}$ к моноядерным комплексам [7]. Существует интерес в расширении возможностей подобных комплексов присоединять дополнительные атомы металлов, т.е. образовывать соединения большей нуклеарности. Одним из таких способов является введение в комплексы лигандов, например дифенилфосфиновых [8], которые способны дополнительно координировать атомы других металлов. 
Ранее [9] нами был предложен метод получения биядерных $\mu$-фенилвинилиденовых комплексов типа $\mathrm{Cp}(\mathrm{CO})_{2} \operatorname{MnPt}(\mu-\mathrm{C}=\mathrm{CHPh})\left(\mathrm{PPh}_{2} \mathrm{H}\right)(\mathrm{L}) \quad\left[\mathrm{L}=\mathrm{PPh}_{3}, \mathrm{P}\left(\mathrm{OPr}^{\mathrm{i}}\right)_{3}\right]$, содержащих в координационной сфере атома платины дифенилфосфиновый $\mathrm{PPh}_{2} \mathrm{H}$ лиганд. В настоящей работе этот подход распространен на комплексы с RePt металлоостовом, синтезированы соответствующие комплексы $\mathrm{Cp}(\mathrm{CO})_{2} \operatorname{RePt}(\mu-\mathrm{C}=\mathrm{CHPh})\left(\mathrm{PPh}_{2} \mathrm{H}\right)(\mathrm{L})\left[\mathrm{L}=\mathrm{PPh}_{3}(\mathbf{1}), \mathrm{P}\left(\mathrm{OPr}^{\mathrm{i}}\right)_{3}\right.$ (2)], изучены их физико-химические свойства методами ИК, ЯМР-спектроскопии и электрохимии.

\section{Экспериментальная часть}

Все операции по синтезу и выделению комплексов проводили в атмосфере аргона. В работе использовали растворители (бензол, диэтиловый эфир, петролейный эфир, хлористый метилен), предварительно очищенные от примесей, следов воды и кислорода перегонкой в инертной атмосфере над соответствующими осушителями. Исходные соединения $\mathrm{Cp}(\mathrm{CO})_{2} \operatorname{RePt}(\mu-\mathrm{C}=\mathrm{CHPh})(\mathrm{CO})(\mathrm{L})\left(\mathrm{L}=\mathrm{PPh}_{3}, \mathrm{P}\left(\mathrm{OPr}^{\mathrm{i}}\right)_{3}\right.$ синтезировали по методикам [10, 11$]$.

Измерение спектров ИК проводили на ИК Фурье-спектрометре Shimadzu IRTracer-100 (Япония). Регистрация спектров ЯМР ${ }^{1} \mathrm{H},{ }^{13} \mathrm{C}$ и ${ }^{31} \mathrm{P}$ выполняли на Фурье-спектрометре ЯМР AVANCEIII 600 (Bruker, Германия). Химические сдвиги в спектрах определены относительно остаточных протонов дейтерированных растворителей и приведены в миллионных долях (м.д.). Константы спин-спиновых взаимодействий указаны в герцах (Гц).

Комплексы $\mathrm{Cp}(\mathrm{CO})_{2} \operatorname{RePt}(\mu-\mathrm{C}=\mathrm{CHPh})\left(\mathrm{PPh}_{2} \mathrm{H}\right)(\mathrm{L})\left[\mathrm{L}=\mathrm{PPh}_{3}(\mathbf{1}), \mathrm{P}\left(\mathrm{OPr}^{\mathrm{i}}\right)_{3}\right.$ (2)] синтезировали по следующей общей методике:

К раствору 0.100 ммоль $\mathrm{Cp}(\mathrm{CO})_{2} \operatorname{RePt}(\mu-\mathrm{C}=\mathrm{CHPh})(\mathrm{L})(\mathrm{CO})$ в 2 мл бензола прибавляли стехиометрическое количество 18 мкл (0.104 ммоль) $\mathrm{Ph}_{2} \mathrm{PH}$. Полученный раствор перемешивали 5 мин и сконцентрировали в вакууме. Остаток растворяли в минимальном количестве смеси (бензол/гексан $=1 / 2)$ и наносили на хроматографическую колонку $\mathrm{Al}_{2} \mathrm{O}_{3}$ $(\mathrm{d}=2 \mathrm{~cm}, \mathrm{~L}=12 \mathrm{~cm})$.

Комплекс $\mathrm{Cp}(\mathrm{CO})_{2} \operatorname{RePt}(\mu-\mathrm{C}=\mathrm{CHPh})\left(\mathrm{PPh}_{2} \mathrm{H}\right)\left(\mathrm{PPh}_{3}\right)(\mathbf{1})$

Широкую светло-желтую зону элюировали бензолом. После удаления растворителя при пониженном давлении получено 100 мг (95\%) мелкокристаллического комплекса 1.

Найдено: C, 52.24; Н, 3.62\%. Вычислено $\left(\mathrm{C}_{45} \mathrm{H}_{37} \mathrm{O}_{2} \mathrm{P}_{2} \mathrm{PtRe}, 1053.02\right)$ : C, 51.33; H, 3.54\%.

ИК-спектр $\left(\mathrm{CH}_{2} \mathrm{Cl}_{2}, \nu \mathrm{CO} \mathrm{cm}^{-1}\right): 1930,1858$.

Изомер 1а

${ }^{1} \mathrm{H}$ ЯMР $\left(\mathrm{CD}_{2} \mathrm{Cl}_{2}, 25{ }^{\circ} \mathrm{C}\right) \delta, J$ Гц: 4,86 (c, $\left.\mathrm{C}_{5} \underline{H}_{5}\right) ; 5,74$ (ддд ${ }^{1}, J_{\mathrm{HP}}=346,{ }^{3} J_{\mathrm{HP}}=4,8,{ }^{2} J_{\mathrm{HPt}}=9,7$, $\left.\mathrm{Ph}_{2} \mathrm{P} \underline{H}\right) ; 6,96-7,65\left(\underline{P h_{2}} \mathrm{PH}, \underline{P h_{3} \mathrm{P}},=\mathrm{C}^{2} \mathrm{H} \underline{P h}\right) ; 7,79$ (ддд, $\left.{ }^{4} J_{\mathrm{PH}}=23,6,{ }^{4} J_{\mathrm{PH}}=14,2,{ }^{3} J_{\mathrm{PHH}}=17,8,=\mathrm{C}^{2} \underline{H}\right)$;

${ }^{31} \mathrm{P}\left\{{ }^{1} \mathrm{H}\right\}$ ЯМР $\left(\mathrm{CD}_{2} \mathrm{Cl}_{2}, 25^{\circ} \mathrm{C}\right) \delta, J$ Гц: 37,91 (дд, $\left.J_{\mathrm{PP}}=25,0, J_{\mathrm{PtP}}=4494, \underline{P} \mathrm{Ph}_{3}\right) ; 6,49$ (дд, $J_{\mathrm{PP}}=25,0$, $\left.J_{\mathrm{PtP}}=2428, \mathrm{H} \underline{P} \mathrm{Ph}_{2}\right)$.

Изомер 16

${ }^{1} \mathrm{H}$ ЯMР $\left(\mathrm{CD}_{2} \mathrm{Cl}_{2}, 25{ }^{\circ} \mathrm{C}\right) \delta, J$ Гц: $5,01\left(\mathrm{c}, \mathrm{C}_{5} \underline{H}_{5}\right) ; 6,37$ (ддд, $J_{\mathrm{HP}}=363,{ }^{3} J_{\mathrm{HP}}=10,3,{ }^{2} J_{\mathrm{HPt}}=14,7$,

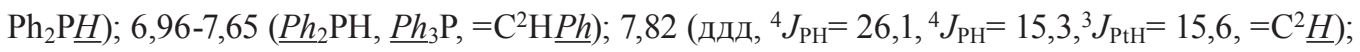

${ }^{31} \mathrm{P}\left\{{ }^{1} \mathrm{H}\right\}$ ЯМР $\left(\mathrm{CD}_{2} \mathrm{Cl}_{2}, 25{ }^{\circ} \mathrm{C}\right) \delta, J$ Гц: $\delta: 27,80$ (дд, $\left.J_{\mathrm{PP}}=22,9, J_{\mathrm{PtP}}=2514, \underline{P} \mathrm{Ph}_{3}\right) ; 12,13$ (дд, $J_{\mathrm{PP}}=$ 22,9, $\left.J_{\mathrm{PtP}}=4386, \mathrm{H} \underline{P} \mathrm{Ph}_{2}\right)$.

Комплекс $\mathrm{Cp}(\mathrm{CO})_{2} \operatorname{RePt}(\mu-\mathrm{C}=\mathrm{CHPh})\left(\mathrm{PPh}_{2} \mathrm{H}\right)\left[\mathrm{P}\left(\mathrm{OPr}^{\mathrm{i}}\right)_{3}\right](2)$ с выходом $92 \%$

ддд - дублет дублет дублетов.

$$
-387-
$$


Широкую зону ярко-желтого цвета элюировали смесью гексан/бензолом (1/4). После удаления растворителей при пониженном давлении получено 90 мг (90\%) комплекса 2 в виде мелких кристаллов оранжевого цвета.

Найдено: С, 44.30; H, 4.52\%. Вычислено ( $\left.\mathrm{C}_{36} \mathrm{H}_{43} \mathrm{O}_{5} \mathrm{P}_{2} \mathrm{PtRe}, 998.97\right)$ : C, 43.28; H, 4.34\%.

ИК-спектр $\left(\mathrm{CH}_{2} \mathrm{Cl}_{2}, v(\mathrm{CO}) \mathrm{cm}^{-1}\right): 1931,1855$.

${ }^{1} \mathrm{H}$ ЯМР $\left(\mathrm{CD}_{2} \mathrm{Cl}_{2}, 25{ }^{\circ} \mathrm{C}\right) \delta, J$ Гц: 1,22 (д, $\left.{ }^{3} J_{\mathrm{HH}}=6,27,-\underline{\mathrm{H}}_{3}\right) ; 4,82$ (м, - $\left.\underline{\mathrm{H}}\right) ; 4,99$ (с, $\left.\mathrm{C}_{5} \underline{H}_{5}\right) ; 6,84$ (ддд, $\left.J_{\mathrm{HP}}=352,{ }^{3} J_{\mathrm{HP}}=6,27,{ }^{2} J_{\mathrm{HPt}}=14,1, \mathrm{Ph}_{2} \mathrm{P} \underline{H}\right) ; 7,10-7,70\left(\underline{P h_{2}} \mathrm{PH},=\mathrm{C}^{2} \mathrm{H} \underline{P h}\right) ; 8,47\left(\right.$ дд, ${ }^{4} J_{\mathrm{PH}} \sim^{4} J_{\mathrm{PH}}=16,2$, $\left.=\mathrm{C}^{2} \underline{H}\right)$;

${ }^{31} \mathrm{P}\left\{{ }^{1} \mathrm{H}\right\}$ ЯМР $\left(\mathrm{CD}_{2} \mathrm{Cl}_{2}, 25^{\circ} \mathrm{C}\right) \delta, J$ Гц: 131,00 (дд, $\left.J_{\mathrm{PP}}=2,7, J_{\mathrm{PtP}}=7005 \underline{P}\left(\mathrm{OPr}^{\mathrm{i}}\right)_{3}\right) ; 4,06$ (дд, $J_{\mathrm{PP}}=$ $25,0, J_{\mathrm{PtP}}=2186, \mathrm{HPPh}$ );

${ }^{13} \mathrm{C}\left\{{ }^{1} \mathrm{H}\right\}$ ЯMP $\left(\mathrm{CD}_{2} \mathrm{Cl}_{2}, 25{ }^{\circ} \mathrm{C}\right) \delta, J$ Гц: 23,6 (д, $\left.{ }^{3} J_{\mathrm{CP}}=4,6,-\underline{C} \mathrm{H}_{3}\right) ; 69,8$ (д, $\left.{ }^{3} J_{\mathrm{CPt}}=24,1,-\underline{C} \mathrm{H}\right) ; 85,3$ (c, $\left.\underline{C}_{5} \mathrm{H}_{5}\right) ; 123,6-129,6\left(\underline{P h_{2}} \mathrm{PH},=\mathrm{C}^{2} \mathrm{H} \underline{P h}\right) ; 137,3$ (ддд, $\left.{ }^{3} J_{\mathrm{CP}} \approx^{3} J_{\mathrm{PH}}=3,3,{ }^{2} J_{\mathrm{CPt}}=119,7,=\underline{C}^{2} \mathrm{HPh}\right) ; 143,4$ (дд, ${ }^{4} J_{\mathrm{PP}}=10,4,{ }^{4} J_{\mathrm{PP}}=15,6, \underline{C}_{\text {кл }}=\mathrm{C}^{2} \mathrm{H} \underline{P h}$ ); 228,1 (ддд, ${ }^{2} J_{\mathrm{CP}}=6,5,{ }^{2} J_{\mathrm{CP}}=64,4, J_{\mathrm{CPt}}=827, \mu-\underline{C}^{1}=$ ).

Электрохимические измерения проводили с использованием свежеприготовленных растворов соединений в очищенном ацетонитриле, с добавлением $0.1 \mathrm{M} \mathrm{Et}_{4} \mathrm{NBF}_{4}$ в качестве фонового электролита, в атмосфере аргона при комнатной температуре. Полярограммы и циклические вольтамперограммы (ЦВА) регистрировали на потенциостате IPC-Pro М (ООО НТФ «Вольта», Санкт-Петербург, Россия). В качестве рабочих электродов ${ }^{2}$ использовали ртутный капельный электрод с принудительным отрывом капель $(\mathrm{m}=3.6 \mathrm{мг} / \mathrm{c}, \mathrm{t}=0.23 \mathrm{c})$ и стационарный: платиновый (d = 1 мм) или стеклоуглеродный (d=5 мм) электрод, в тефлоновых корпусах $\left(\mathrm{d}=10\right.$ мм). Электродом сравнения служил полуэлемент $\mathrm{Ag} / 0.1 \mathrm{M} \mathrm{AgNO}_{3}$ в $\mathrm{MeCN}$, соединенный с ячейкой электролитическим мостом, заполненным фоновым электролитом, через капилляр Луггина, вспомогательным - платиновая спираль, помещенная в стеклянную трубку с пористым фильтром. Число электронов, участвующих в каждой редокс-стадии, определяли сравнением высот волн исследуемых соединений с высотой одноэлектронной волны хорошо изученного комплекса $\mathrm{Cp}(\mathrm{CO})_{2} \mathrm{Re}=\mathrm{C}=\mathrm{CHPh}[12]$. Электролиз при контролируемом потенциале (ЭКП) проводили на потенциостате IPC-Pro M (рабочий электрод - платиновая пластина площадью $4 \mathrm{~cm}^{2}$, электрод сравнения - $\mathrm{Ag} / 0.1 \mathrm{M} \mathrm{AgNO}_{3}$ в $\mathrm{MeCN}$, вспомогательный $-\mathrm{Pt}$ ) при интенсивном барботировании аргоном. Для химического окисления использовали ферроцения борфторид $[\mathrm{Fc}]\left[\mathrm{BF}_{4}\right]\left(\mathrm{Fc}=\left[\left(\mathrm{C}_{5} \mathrm{H}_{5}\right)_{2} \mathrm{Fe}\right]^{+}\right)[13]$.

\section{Результаты и обсуждение}

Подобно MnPt-соединениям [9], комплексы $\mathrm{Cp}(\mathrm{CO})_{2} \operatorname{RePt}(\mu-\mathrm{C}=\mathrm{CHPh})(\mathrm{L})_{2}\left[\mathrm{~L}=\mathrm{PPh}_{3}\right.$ (3), $\left.\mathrm{P}\left(\mathrm{OPr}^{\mathrm{i}}\right)_{3}(4)\right]$ не способны кпрямому взаимодействию с дифенилфосфином. Поэтому была предложена двухстадийная схема получения биядерных $\mu$-винилиденовых комплексов $\mathrm{Cp}(\mathrm{CO})_{2} \operatorname{RePt}(\mu-$ $\mathrm{C}=\mathrm{CHPh})\left(\mathrm{PPh}_{2} \mathrm{H}\right)(\mathrm{L})\left[\mathrm{L}=\mathrm{PPh}_{3}(\mathbf{1}), \mathrm{P}\left(\mathrm{OPr}^{\mathrm{i}}\right)_{3}(\mathbf{2})\right]$, включающая:

1) карбонилирование соединений $\mathrm{Cp}(\mathrm{CO})_{2} \operatorname{RePt}(\mu-\mathrm{C}=\mathrm{CHPh})(\mathrm{L})_{2}\left[\mathrm{~L}=\mathrm{PPh}_{3}(\mathbf{3}), \mathrm{P}\left(\mathrm{OPr}^{\mathrm{i}}\right)_{3}\right.$ (4)] с помощью $\mathrm{Co}_{2}(\mathrm{CO})_{8}$, с образованием комплексов $\mathrm{Cp}(\mathrm{CO})_{2} \operatorname{RePt}(\mu-\mathrm{C}=\mathrm{CHPh})(\mathrm{CO})(\mathrm{L})\left[\mathrm{L}=\mathrm{PPh}_{3}(\mathbf{5})\right.$ [2], $\left.\mathrm{P}\left(\mathrm{OPr}^{\mathrm{i}}\right)_{3}(6)\right]$;

2 Использование различных рабочих электродов позволяет исследовать процессы окисления и восстановления соединений в более широкой области потенциалов. Так, рабочая область измерения потенциалов в ацетонитриле (относительно $\mathrm{Ag} / 0.1 \mathrm{M} \mathrm{AgNO}_{3}$ в $\mathrm{MeCN}$ ) на р.к.э. находится в пределах от 0,30 до -3,20 B, на Pt и CУ электродах от 2,00 до -2,20 В и от 2,00 до -2,60 В соответственно. 


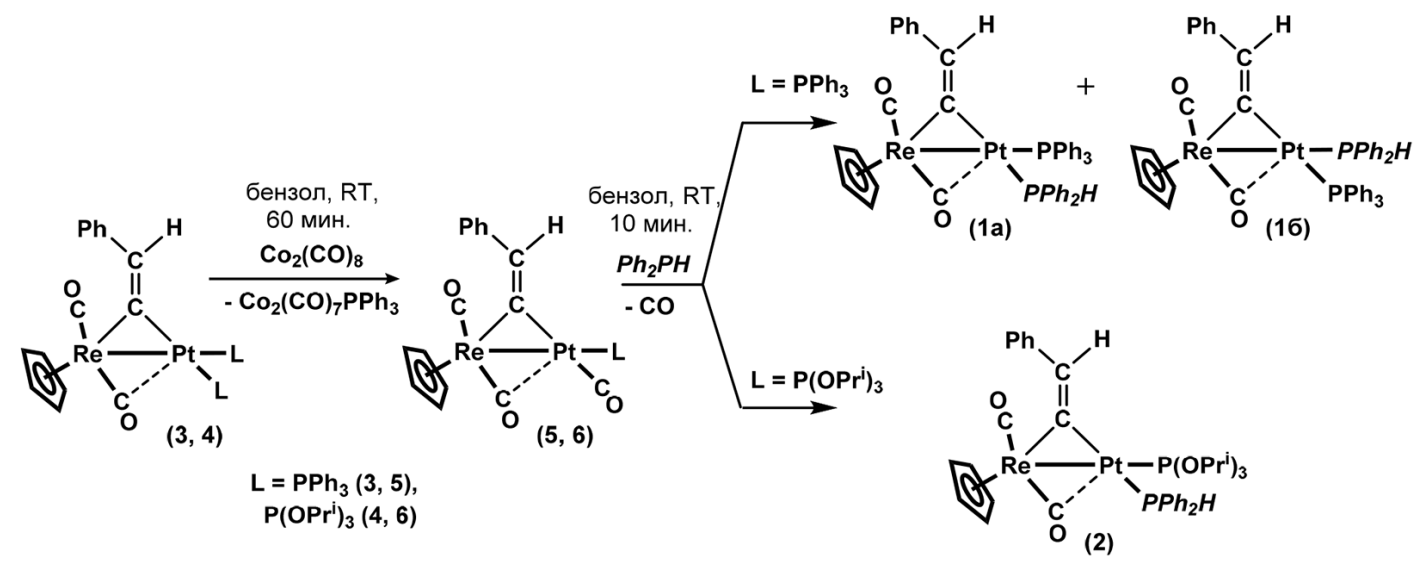

Рис. 1. Схема синтеза $\mathrm{Cp}(\mathrm{CO})_{2} \operatorname{RePt}(\mu-\mathrm{C}=\mathrm{CHPh})\left(\mathrm{PPh}_{2} \mathrm{H}\right)(\mathrm{L})\left[\mathrm{L}=\mathrm{PPh}_{3}(\mathbf{1}), \mathrm{P}\left(\mathrm{OPr}^{\mathrm{i}}\right)_{3}(\mathbf{2})\right]$

Fig. 1. Scheme of $\mathrm{Cp}(\mathrm{CO})_{2} \operatorname{RePt}(\mu-\mathrm{C}=\mathrm{CHPh})\left(\mathrm{PPh}_{2} \mathrm{H}\right)(\mathrm{L})\left[\mathrm{L}=\mathrm{PPh}_{3}(\mathbf{1}), \mathrm{P}\left(\mathrm{OPr}^{\mathrm{i}}\right)_{3}(\mathbf{2})\right]$ synthesis

2) замещение карбонильного лиганда при атоме платины в полученных на первой стадии комплексах 5 и 6 на дифенилфосфин $\mathrm{Ph}_{2} \mathrm{PH}$ (рис. 1).

Наибольшие выходы, 90 - 95\%, промежуточных и целевых продуктов достигаются при проведении реакций в бензоле при комнатной температуре. Для выделения комплексов 1, 2, 5 и 6 использовали метод колоночной хроматографии.

Комплексы 1 и 2 изучены методами ЯМР- и ИК-спектроскопии. Их строение предложено на основании спектров ЯМР ${ }^{1} \mathrm{H}$ и ${ }^{31} \mathrm{P}$, содержащих характеристичные сигналы фенилвинилиденового и дифенилфосфинового лигандов (табл. 1). В спектре ПМР комплекса 2 сигналы протона винилидена $\mu-\mathrm{C}=\mathrm{C}(\mathrm{Ph}) H$ и дифенилфосфина $\mathrm{Ph}_{2} \mathrm{PH}$ находятся при 8.47 и 6.84 м.д. $\left(J_{\mathrm{PH}}=352\right.$ Гц, ${ }^{3} J_{\mathrm{PH}}=6.27$ Гц ${ }^{3},{ }^{2} J_{\mathrm{PtH}}=14.1$ Гц). Однако для 1 наблюдаются два набора сигналов протона фенилвинилидена (7.83 и 7.78 м.д.) и протона лиганда $\mathrm{Ph}_{2} \mathrm{P}-H\left[\delta 5.74\right.$ м.д. $\left(J_{\mathrm{PH}}=346\right.$ Гц, ${ }^{3} J_{\mathrm{PH}}=4.68$ Гц $)$ и 6.37 м.д. $\left(J_{\mathrm{PH}}=364\right.$ Гц, ${ }^{3} J_{\mathrm{PH}}=9.9$ Гц)], свидетельствующих о том, что этот комплекс в растворах существует в виде двух изомеров. На основании данных спектров ЯМР ${ }^{31} \mathrm{P}$ установлено, что изомеры комплекса 1 отличаются различным расположением фосфорных лигандов при атоме $\mathrm{Pt}$ относительно фрагмента $\left[\mathrm{Cp}(\mathrm{CO})_{2} \mathrm{Re}\right]$ и винилиденового лиганда и находятся в соотношении 1:1.8. В спектрах ЯМР ${ }^{31} \mathrm{P}$ сигналы ядер атома фосфора дифенилфосфинового лиганда при 6.49 м.д. (комплекс 1a) и 4.75 м.д. (комплекс 2) содержат константы спин-спинового взаимодействия $J_{\mathrm{PtP}} 2428$ и 2195 Гц соответственно, что свидетельствует о цис-положении лиганда $\mathrm{Ph}_{2} \mathrm{PH}$ относительно атома $\operatorname{Re}[14,15]$. Однако в изомерном комплексе 1 б значения констант $J_{\mathrm{PtP}}$, равные 2514 Гц для $\mathrm{PPh}_{3}$ лиганда при 27.80 м.д. и 4386 Гц для $\mathrm{Ph}_{2} \mathrm{PH}$ при 12.13 м.д., указывают на транс-расположение лиганда $\mathrm{Ph}_{2} \mathrm{PH}$. Значения констант $J_{\mathrm{PtP}}$ сигналов, отвечающих трансрасположенным лигандам $\mathrm{PPh}_{3}(\mathbf{1 a})$, при 37.91 м.д. и $\mathrm{P}\left(\mathrm{OPr}^{\mathrm{i}}\right)_{3}(\mathbf{2})$ при 130.62 м.д. составляют 4494 и 7058 Гц соответственно (табл. 1).

ИК-спектры комплексов 1 и 2 в области валентных колебании СО-групп содержат две полосы: высокочастотную при $1930 \mathrm{~cm}^{-1}$ (1), $1931 \mathrm{~cm}^{-1}$ (2), соответствующую терминальной СОгруппе при атоме рения, и низкочастотную при $1858 \mathrm{~cm}^{-1}(\mathbf{1}), 1855 \mathrm{~cm}^{-1}$ (2), относящуюся ко второй карбонильной группе при атоме рения, которая участвует в полумостиковом взаимодействии 
Таблица 1. Данные спектров ЯМР ${ }^{1} \mathrm{H},{ }^{31} \mathrm{P}$ и ${ }^{13} \mathrm{C}\left(\delta\right.$, м.Д., [J, Гц], $\left.\mathrm{CD}_{2} \mathrm{Cl}_{2}\right)$ комплексов 1 и 2

Table 1. NMR ${ }^{1} \mathrm{H},{ }^{31} \mathrm{P}$ and ${ }^{13} \mathrm{C}\left(\delta, \mathrm{ppm},[J, \mathrm{~Hz}], \mathrm{CD}_{2} \mathrm{Cl}_{2}\right)$ spectroscopic data of $\mathbf{1}$ and $\mathbf{2}$

\begin{tabular}{|c|c|c|c|c|c|c|c|c|}
\hline \multirow{4}{*}{ № } & \multicolumn{8}{|c|}{ ЯМР (d, м.Д.; J, Гц) } \\
\hline & \multicolumn{3}{|c|}{${ }^{1} \mathrm{H}$} & \multicolumn{3}{|c|}{${ }^{13} \mathrm{C}$} & \multicolumn{2}{|c|}{${ }^{31} \mathrm{P}$} \\
\hline & \multirow{2}{*}{$=\mathrm{CH}$} & \multirow{2}{*}{$\mathrm{C}_{5} H_{5}$} & \multirow{2}{*}{$\mathrm{PPh}_{2} H$} & \multicolumn{2}{|c|}{$\mu-\mathrm{C}^{1}=\mathrm{C}^{2} \mathrm{HPh}$} & \multirow{2}{*}{$C_{5} \mathrm{H}_{5}$} & \multirow{2}{*}{$\begin{array}{c}\mathrm{PPh}_{3} / \\
P\left(\mathrm{OPr}^{\mathrm{i}}\right)_{3}\end{array}$} & \multirow{2}{*}{$\mathrm{PPh}_{2} \mathrm{H}$} \\
\hline & & & & $C^{1}=$ & $=C^{2}$ & & & \\
\hline 1a & $\begin{array}{l}7,79 \text { ддд } \\
{ }^{4} J_{\mathrm{PH}}=23,6 \\
{ }^{4} J_{\mathrm{PH}}=14,2 \\
{ }^{3} J_{\mathrm{PtH}}=17,8\end{array}$ & $4,86 \mathrm{c}$ & $\begin{array}{l}5,74 \text { ддд } \\
J_{\mathrm{HP}}=346 \\
{ }^{3} J_{\mathrm{HP}}=4,8 \\
{ }^{2} J_{\mathrm{HPt}}=9,7\end{array}$ & $\begin{array}{l}232,2 \text { д } \\
{ }^{2} J_{\mathrm{CP}}=65,0\end{array}$ & $\begin{array}{l}138,4 \text { дд } \\
{ }^{2} J_{\mathrm{CPt}}=103,4\end{array}$ & $85.6 \mathrm{c}$ & $\begin{array}{l}37.91 \text { дд } \\
J_{\mathrm{PP}}=25,0 \\
J_{\mathrm{PtP}}=4494 \\
\mathrm{PPh}_{3}\end{array}$ & $\begin{array}{l}6,49 \text { дд } \\
J_{\mathrm{PP}}=25,0 \\
J_{\mathrm{PtP}}=2428\end{array}$ \\
\hline 10 & $\begin{array}{l}7,82 \text { ддд } \\
{ }^{4} J_{\mathrm{PH}}=26,1 \\
{ }^{4} J_{\mathrm{PH}}=15,3 \\
{ }^{3} J_{\mathrm{PtH}}=15,6\end{array}$ & $5,01 \mathrm{c}$ & $\begin{array}{l}6,37 \text { ддд } \\
J_{\mathrm{HP}}=363 \\
{ }^{3} J_{\mathrm{HP}}=10,3 \\
{ }^{2} J_{\mathrm{HPt}}=14,7\end{array}$ & $230,5 \mathrm{c}$ & $\begin{array}{l}136,8 \text { дд } \\
{ }^{2} J_{\mathrm{CPt}}=115,1\end{array}$ & $85.5 \mathrm{c}$ & $\begin{array}{l}27,80 \text { дд } \\
J_{\mathrm{PP}}=22,9 \\
J_{\mathrm{PtP}}=2514 \\
\mathrm{PPh}_{3}\end{array}$ & $\begin{array}{l}12,13 \text { дд } \\
J_{\mathrm{PP}}=22,9 \\
J_{\mathrm{PtP}}=4386\end{array}$ \\
\hline 2 & $\begin{array}{l}8,47 \text { дд } \\
{ }^{4} J_{\mathrm{PH}}{ }^{4} J_{\mathrm{PH}}=16,2\end{array}$ & $4,99 \mathrm{c}$ & $\begin{array}{l}6,84 \text { ддд } \\
J_{\mathrm{HP}}=352 \\
{ }^{3} J_{\mathrm{HP}}=6,27 \\
{ }^{2} J_{\mathrm{HPt}}=14,1\end{array}$ & $\begin{array}{l}228,1 \text { ддд } \\
{ }^{2} J_{\mathrm{CP}}=6,5 \\
{ }^{2} J_{\mathrm{CP}}=64,4 \\
J_{\mathrm{CPt}}=827\end{array}$ & $\begin{array}{l}137,3 \text { ддд } \\
{ }^{3} J_{\mathrm{CP}} »^{3} J_{\mathrm{PH}}=3,3 \\
{ }^{2} J_{\mathrm{CPt}}=119,7\end{array}$ & $85,3 \mathrm{c}$ & $\begin{array}{l}131,00 \text { дд } \\
J_{\mathrm{PP}}=2,7 \\
J_{\mathrm{PtP}}=7005 \\
\mathrm{P}\left(\mathrm{OPr}^{\mathrm{i}}\right)_{3}\end{array}$ & $\begin{array}{l}4,06 \text { дд } \\
J_{\mathrm{PP}}=25,0 \\
J_{\mathrm{PtP}}=2186\end{array}$ \\
\hline
\end{tabular}

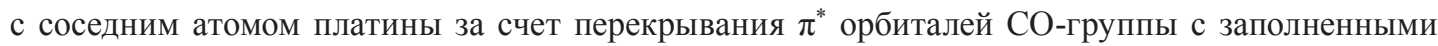
d-орбиталями платины. При этом, в отличие от изученных ранее MnPt комплексов [9], в RePtкомплексах введение дифенилфосфинового лиганда в координационную сферу атома платины практически не влияет на изменение степени взаимодействия $\mathrm{Pt} \rightarrow \mathrm{CO}_{\text {пм }}$. Так, величина $\Delta v(\mathrm{CO})$, характеризующая степень полумостикового взаимодействия и определяющаяся разностью частот валентных колебаний терминальной и полумостиковой СО-групп [16], практически не меняется при переходе от комплексов $\mathrm{Cp}(\mathrm{CO})_{2} \operatorname{RePt}(\mu-\mathrm{C}=\mathrm{CHPh})\left(\mathrm{PPh}_{3}\right)_{2}(\mathbf{3})\left(\Delta v(\mathrm{CO})=75 \mathrm{~cm}^{-1}\right)$ и $\mathrm{Cp}(\mathrm{CO})_{2} \operatorname{RePt}(\mu-\mathrm{C}=\mathrm{CHPh})\left[\mathrm{P}\left(\mathrm{OPr}^{\mathrm{i}}\right)_{3}\right]\left(\mathrm{PPh}_{3}\right)\left(\Delta v(\mathrm{CO})=76 \mathrm{~cm}^{-1}\right)$ к 1 и $2\left(\Delta v(\mathrm{CO})=72\right.$ и $\left.76 \mathrm{~cm}^{-1}\right)$ соответственно, тогда как ранее для MnPt-комплексов введение дифенилфосфиновых лигандов приводило к сдвигу $v(\mathrm{CO})_{\text {пм }}$ в низкочастотную область на 10 и $14 \mathrm{~cm}^{-1}$ относительно значений исходных комплексов и, соответственно, к усилению полумостикового взаимодействия [9] (табл. 2).

Редокс-свойства новых комплексов 1 и 2 исследованы методами циклической вольтамперометрии на $\mathrm{Pt}$ и СУ электродах, полярографии на р.к.э. и электролиза при контролируемом

Таблица 2. Данные ИК-спектров комплексов $\mathrm{Cp}(\mathrm{CO})_{2} \operatorname{RePt}(\mu-\mathrm{C}=\mathrm{CHPh})(\mathrm{L})\left(\mathrm{L}^{\prime}\right)$

Table 2. IR data for the $\mathrm{Cp}(\mathrm{CO})_{2} \operatorname{RePt}(\mu-\mathrm{C}=\mathrm{CHPh})(\mathrm{L})(\mathrm{L} ')$

\begin{tabular}{lll}
\hline \multicolumn{1}{c}{ Комплекс } & \multicolumn{1}{c}{$v(\mathrm{CO}), \mathrm{cm}^{-1}\left(\right.$ в р-ре $\left.\mathrm{CH}_{2} \mathrm{Cl}_{2}\right)$} & $\Delta v(\mathrm{CO})$ \\
\hline $\mathrm{Cp}(\mathrm{CO})_{2} \operatorname{RePt}(\mu-\mathrm{C}=\mathrm{CHPh})\left(\mathrm{PPh}_{2} \mathrm{H}\right)\left(\mathrm{PPh}_{3}\right)(\mathbf{1})$ & 1930,1858 & 72 \\
\hline $\mathrm{Cp}(\mathrm{CO})_{2} \operatorname{RePt}(\mu-\mathrm{C}=\mathrm{CHPh})\left(\mathrm{PPh}_{3}\right)_{2}(\mathbf{3})$ & 1933,1858 & 75 \\
\hline $\mathrm{Cp}(\mathrm{CO})_{2} \operatorname{RePt}(\mu-\mathrm{C}=\mathrm{CHPh})(\mathrm{CO})\left(\mathrm{PPh}_{3}\right)(\mathbf{5})$ & $2030,1941,1876$ & 63 \\
\hline $\mathrm{Cp}(\mathrm{CO})_{2} \operatorname{RePt}(\mu-\mathrm{C}=\mathrm{CHPh})\left(\mathrm{PPh}_{2} \mathrm{H}\right)\left[\mathrm{P}(\mathrm{OPr})_{3}\right](\mathbf{2})$ & 1931,1855 & 76 \\
\hline $\mathrm{Cp}(\mathrm{CO})_{2} \operatorname{RePt}(\mu-\mathrm{C}=\mathrm{CHPh})\left[\mathrm{P}(\mathrm{OPr})_{3}\right]_{2}(\mathbf{4})$ & 1943,1873 & 70 \\
\hline $\mathrm{Cp}(\mathrm{CO})_{2} \operatorname{RePt}(\mu-\mathrm{C}=\mathrm{CHPh})(\mathrm{CO})\left[\mathrm{P}\left(\mathrm{OPr}^{\mathrm{i}}\right)_{3}\right](\mathbf{6})$ & $2030,1940,1878$ & 62 \\
\hline
\end{tabular}


потенциале в ацетонитриле. Электрохимические характеристики 1 и $\mathbf{2}$, а также ранее изученных биядерных 3, 4 [11] и моноядерного $\mathrm{Cp}(\mathrm{CO})_{2} \mathrm{Re}=\mathrm{C}=\mathrm{CHPh}$ (7) [12] фенилвинилиденовых комплексов приведены в табл. 3, ЦВА соединений 2 и 7 на Рt электроде - на рис. 2.

Комплексы 1 и 2 окисляются на Pt и СУ электродах и восстанавливаются на р.к.э. в две необратимые стадии (табл. 3). Сравнение значений потенциалов первых стадий окисления и восстановления комплексов 1,2 с 3, 4 показывает, что замена одного из лигандов $\mathrm{PPh}_{3}$ или

Таблица 3. Электрохимические характеристики фенилвинилиденовых комплексов рения (MeCN, $0.1 \mathrm{M} \mathrm{Et}_{4} \mathrm{NBF}_{4}, 2 \mathrm{мM}, \mathrm{Ag} / 0.1 \mathrm{M} \mathrm{AgNO}_{3}$ в $\mathrm{MeCN}$ )

Table 3. Electrochemical characteristics of the phenylvinylidene rhenium complexes $\left(\mathrm{MeCN}, 0.1 \mathrm{M} \mathrm{Et}_{4} \mathrm{NBF}_{4}\right.$, $2 \mathrm{MM}, \mathrm{Ag} / 0.1 \mathrm{M} \mathrm{AgNO}_{3}$ in $\mathrm{MeCN}$ )

\begin{tabular}{|c|c|c|c|}
\hline \multirow{3}{*}{ Соединение } & \multicolumn{3}{|c|}{$\mathrm{E}_{1 / 2}, \mathrm{~B}(\mathrm{n})$} \\
\hline & \multicolumn{2}{|c|}{ Окисление } & \multirow{2}{*}{$\frac{\text { Восстановление }}{\text { Р.к.э. }}$} \\
\hline & $\mathrm{Pt}$ & $\mathrm{CY}$ & \\
\hline \multirow{2}{*}{$\mathrm{Cp}(\mathrm{CO})_{2} \operatorname{RePt}(\mu-\mathrm{C}=\mathrm{CHPh})\left(\mathrm{PPh}_{2} \mathrm{H}\right)\left(\mathrm{PPh}_{3}\right)(\mathbf{1})$} & $-0.09(1)$ & $-0.06(1)$ & $-2.70(1)$ \\
\hline & $0.32(1)$ & $0.38(1)$ & $-2.83(<1)$ \\
\hline \multirow{2}{*}{$\mathrm{Cp}(\mathrm{CO})_{2} \operatorname{RePt}(\mu-\mathrm{C}=\mathrm{CHPh})\left(\mathrm{PPh}_{2} \mathrm{H}\right)\left[\mathrm{P}\left(\mathrm{OPr}^{\mathrm{i}}\right)_{3}\right](\mathbf{2})$} & $-0.14(1)$ & $-0.09(1)$ & $-2.88(1)$ \\
\hline & $0.30(1)$ & $0.40(1)$ & $-3.04(<1)$ \\
\hline \multirow{3}{*}{$\mathrm{Cp}(\mathrm{CO})_{2} \operatorname{RePt}(\mu-\mathrm{C}=\mathrm{CHPh})\left(\mathrm{PPh}_{3}\right)_{2}(\mathbf{3})$} & $-0.07(1)$ & $0.00(1)$ & $-2.60(1)$ \\
\hline & $0.26(<1)$ & $0.29(<1)$ & $-2.80(1)$ \\
\hline & $0.33(<1)$ & $0.36(<1)$ & $-3.00(1)$ \\
\hline \multirow{3}{*}{$\mathrm{Cp}(\mathrm{CO})_{2} \operatorname{RePt}(\mu-\mathrm{C}=\mathrm{CHPh})\left[\mathrm{P}\left(\mathrm{OPr}^{\mathrm{i}}\right)_{3}\right]_{2}(\mathbf{4})$} & $-0.12(1)$ & $-0.07(1)$ & \\
\hline & $0.26(<1)$ & $0.35(<1)$ & $-2.87(1)$ \\
\hline & $0.33(<1)$ & & \\
\hline \multirow{2}{*}{$\mathrm{Cp}(\mathrm{CO})_{2} \mathrm{Re}=\mathrm{C}=\mathrm{CHPh}(7)$} & $0.33(1)$ & $0.36(1)$ & $-2.21(1)$ \\
\hline & & & $-2.82(1)$ \\
\hline
\end{tabular}

Примечание: $\mathrm{n}$ - число электронов, участвующих в электрохимической стадии (знак «<» означает, что высота волны исследуемого соединения меньше, чем высота одноэлектронной волны).

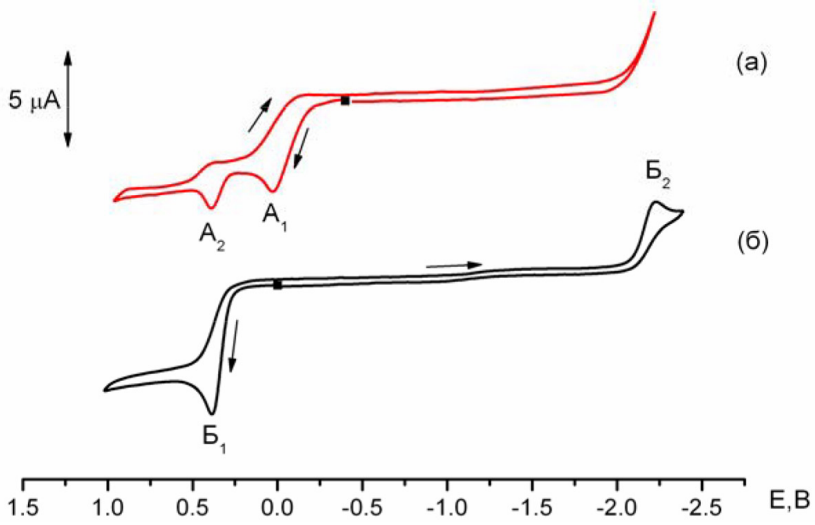

Рис. 2. ЦВАкомплексов: (a) - $\mathrm{Cp}(\mathrm{CO})_{2} \operatorname{RePt}(\mu-\mathrm{C}=\mathrm{CHPh})\left(\mathrm{PPh}_{2} \mathrm{H}\right)\left[\mathrm{P}\left(\mathrm{OPr}^{\mathrm{i}}\right)_{3}\right](\mathbf{2}),\left(\right.$ б) $-\mathrm{Cp}(\mathrm{CO})_{2} \operatorname{Re}=\mathrm{C}=\mathrm{CHPh}(\mathbf{7})$ (Pt, $\mathrm{MeCN}, 0.1 \mathrm{M} \mathrm{Et}_{4} \mathrm{NBF}_{4}, \mathrm{Ag} / 0.1 \mathrm{M} \mathrm{AgNO}_{3}$ в $\mathrm{MeCN}, \mathrm{C}=2 \mathrm{mM}, \mathrm{V}=25 \mathrm{mB} / \mathrm{c}$ )

Fig. 2. Cyclic voltammograms of complexes (a) $-\mathrm{Cp}(\mathrm{CO})_{2} \operatorname{RePt}(\mu-\mathrm{C}=\mathrm{CHPh})\left(\mathrm{PPh}_{2} \mathrm{H}\right)\left[\mathrm{P}\left(\mathrm{OPr}^{\mathrm{i}}\right)_{3}\right]$ (2), (б) $\mathrm{Cp}(\mathrm{CO})_{2} \mathrm{Re}=\mathrm{C}=\mathrm{CHPh}(7)\left(\mathrm{Pt}, \mathrm{MeCN}, 0.1 \mathrm{MEt}_{4} \mathrm{NBF}_{4}, \mathrm{Ag} / 0.1 \mathrm{M} \mathrm{AgNO}_{3}\right.$ in $\mathrm{MeCN}, \mathrm{C}=2 \mathrm{mM}$, scan rate $\left.25 \mathrm{mV} \mathrm{c} \mathrm{c}^{-1}\right)$ 
$\mathrm{P}\left(\mathrm{OPr}^{\mathrm{i}}\right)_{3}$ в 3 и 4 на $\mathrm{PPh}_{2} \mathrm{H}$ приводит к незначительному смещению величин $\mathrm{E}_{1 / 2}$ комплексов 1 и 2 в катодную область, а в случае восстановления 2 и 4 на р.к.э. они практически совпадают. Однако следует отметить, что замена лиганда $\mathrm{PPh}_{3}$ в 1 на $\mathrm{P}\left(\mathrm{OPr}^{\mathrm{i}}\right)_{3}$ также приводит к смещению значений редокс-потенциалов 2 в катодную область (табл. 3), несмотря на то, что в этом случае, согласно электронодонорной способности лигандов $\mathrm{PPh}_{3}$ и $\mathrm{P}\left(\mathrm{OPr}^{\mathrm{i}}\right)_{3}$, должна наблюдаться обратная зависимость. Аналогичное влияние природы фосфорсодержащих лигандов на редокс-потенциалы RePt комплексов выявлено ранее для родственных марганцевых соединений [17].

Значение потенциалов вторых стадий окисления биядерных RePt комплексов 1 и 2 (табл. 3 , рис. $1 a$, пик $\mathrm{A}_{2}$ ) близко к $\mathrm{E}_{1 / 2}$ одноэлектронного окисления моноядерного комплекса $\mathrm{Cp}(\mathrm{CO})_{2} \mathrm{Re}=\mathrm{C}=\mathrm{CHPh}$ (7) (табл. 3, рис. 16, пик $\mathrm{b}_{1}$ ), что свидетельствует о его образовании после одноэлектронного необратимого окисления комплексов $\mathbf{1}$ и $\mathbf{2}$. Для доказательства этого предположения проведен ЭКП предельного тока первой волны окисления комплексов $\mathbf{1}$ и $\mathbf{2}$, а также их химическое окисление с использованием $[\mathrm{Fc}]\left[\mathrm{BF}_{4}\right]$, с последующей идентификацией продуктов окисления комплексов методами электрохимии и ИК-спектроскопии. В ИК-спектрах растворов, отобранных из электрохимической ячейки как после ЭКП, так и после химического окисления комплексов, в области валентных колебаний СО-групп наблюдаются две полосы с частотами (1994 и $\left.1920 \mathrm{~cm}^{-1}\right)$, соответствующими колебаниям карбонильных групп комплекca 7. Следовательно, необратимое одноэлектронное окисление биядерных RePt комплексов 1 и 2 на $\mathrm{Pt}$ и СУ электродах приводит к отщеплению Pt-содержащего фрагмента и образованию моноядерного винилиденового комплекса рения 7:

$$
\begin{aligned}
& \mathrm{Cp}(\mathrm{CO})_{2} \operatorname{RePt}(\mu-\mathrm{C}=\mathrm{CHPh})\left(\mathrm{PPh}_{2} \mathrm{H}\right)(\mathrm{L}) \stackrel{-\mathrm{e}^{-}}{\longrightarrow} \\
& \stackrel{-\mathrm{e}^{-}}{\longrightarrow}\left[\mathrm{Cp}(\mathrm{CO})_{2} \operatorname{RePt}(\mu-\mathrm{C}=\mathrm{CHPh})\left(\mathrm{PPh}_{2} \mathrm{H}\right)(\mathrm{L})\right]^{+\cdot} \rightarrow \\
& \rightarrow \mathrm{Cp}(\mathrm{CO})_{2} \operatorname{Re}=\mathrm{C}=\mathrm{CHPh}+\left[\mathrm{Pt}\left(\mathrm{PPh}_{2} \mathrm{H}\right)\left(\mathrm{L}^{\prime}\right)\right]^{+} \text {solv } \\
& \mathrm{Cp}(\mathrm{CO})_{2} \mathrm{Re}=\mathrm{C}=\mathrm{CHPh} \stackrel{-\mathrm{e}^{-}}{\longrightarrow}\left[\mathrm{Cp}(\mathrm{CO})_{2} \mathrm{Re}=\mathrm{C}=\mathrm{CHPh}\right]^{+} .
\end{aligned}
$$

Таким образом, на примере получения новых $\mu$-фенилвинилиденовых комплексов типа $\mathrm{Cp}(\mathrm{CO})_{2} \operatorname{RePt}(\mu-\mathrm{C}=\mathrm{CHPh})\left(\mathrm{PPh}_{2} \mathrm{H}\right)(\mathrm{L})\left[\mathrm{L}=\mathrm{PPh}_{3}(\mathbf{1}), \mathrm{P}\left(\mathrm{OPr}^{\mathrm{i}}\right)_{3}\right.$ (2)], содержащих дифенилфосфиновые лиганды при атоме платины, показана возможность использования ранее предложенного синтетического подхода [9]. Новые соединения изучены методами ИК и ЯМР спектроскопии и электрохимии. На основании спектроскопических данных предложено строение комплексов $\mathbf{1}$ и 2. Полученные электрохимические данные хорошо согласуются с результатами изучения комплексов методами ИК- и ЯМР-спектроскопии, а также с данными исследования редокссвойств родственных марганцевых соединений [17].

\section{Благодарности / Acknowledgements}

Работа выполнена в рамках государственного задания Института химии и химической технологии СО РАН (проект АААА-А17-117021310221-7) с использованием оборудования Красноярского регионального центра коллективного пользования ФИЦ КНЦ СО РАН.

This work was conducted within the framework of the budget project AAAA-A17-117021310221-7 for Institute of Chemistry and Chemical Technology SB RAS using the equipment of Krasnoyarsk Regional Research Equipment Centre of SB RAS. 


\section{Список литературы / References}

1. Sánchez-Cabrera G., Zuno-Cruz F.J., Rosales-Hoz M.J. Reactivity of alkyne-substituted transition metal clusters: an overview. J. Clust. Sci. 2014. Vol. 25(1), P. 51-82.

2. Buchwalter P., Rosé J., Braunstein P. Multimetallic catalysis based on heterometallic complexes and clusters. Chem. Rev. 2015. Vol. 115, P. 28-126.

3. Böhmer M., Kampert F., Tan T.T.Y., Guisado-Barrios G., Peris E., Hahn F.E. IrIII/AuI and RhIII/AuI heterobimetallic complexes as catalysts for the coupling of nitrobenzene and benzylic alcohol. Organometallics 2018. Vol. 37, P. 4092-4099.

4. Chor B.Y., Koh W.X., Gangully R., Li Y., Chen L., Raja R., Leong W.K. Cobalt-Platinum heterometallic clusters containing N-heterocyclic carbene ligands. J. Organomet. Chem. 2017. Vol. 849-850, P. 48-53.

5. Mohamed A.S., Jourdain I., Knorr M., Boullanger S., Brieger L., Strohmann C. Heterodinuclear diphosphane-bridged Iron-Platinum diyne complexes as metalloligands for the assembly of polymetallic systems (Fe, Pt, Co). J. Clust. Sci. 2019. Vol. 30, P. 1211-1225.

6. Knorr M., Jourdain I. Activation of alkynes by diphosphine- and $\mu$-phosphido-spanned heterobimetallic complexes. Coord. Chem. Rev. 2017. Vol. 350, P. 217-247.

7. Antonova A.B. Use of the $\mathrm{Mn}=\mathrm{C}=\mathrm{C}$ system in organometallic and organic synthesis. Coord. Chem. Rev. 2007. Vol. 251(12), P. 1521-1560.

8. Alonso E., Forniés J., Fortuño C., Martín A., Orpen A.G. Reactivity of a secondary phosphine Platinum(II) complex with [Pt(norbornene) $)_{3}$ and $\mathrm{PPh}_{3}$. Synthesis of new single phosphido-bridged derivatives of Platinum(I) and phosphido-bridged Platinum(II) hydrides. Organometallics 2001. Vol. 20, P. 850-859.

9. Chudin O.S., Verpekin V.V., Kondrasenko A.A., Pavlenko N.I., Rubaylo A.I. Syntheses and physical-chemical properties of phenylvinylidene complexes with $\mathrm{MnPt}$ core, containing diphenylphosphine ligand at the Platinum atom. J. Sib. Fed. Univ. Chem. 2018. Vol. 11(3), P. 390-400.

10. Verpekin V.V., Vasiliev A.D., Kondrasenko A.A., Burmakina G.V., Chudin O.S., Pavlenko N.I., Zimonin D.V., Rubaylo A.I. Chemistry of vinylidene complexes. XXIV. A new $\mu$-vinylidene complex containing RePt core, and platinum-bound carbonyl ligand. Spectroscopic, structural and electrochemical study. J. Mol. Struct. 2018. Vol. 1163, P. 308-315.

11. Chudin O.S., Verpekin V.V., Kondrasenko A.A., Burmakina G.V., Piryazev D.A., Vasiliev A.D., Pavlenko N.I., Zimonin D.V., Rubaylo A.I. Chemistry of vinylidene complexes. XXV. Synthesis and reactions of binuclear $\mu$-vinylidene RePt complexes containing phosphite ligands. Spectroscopic, structural and electrochemical study. Inorganica Chim. Acta. Elsevier S.A. 2020. Vol. 505, P. 119463.

12. Burmakina G.V., Verpekin V.V., Chudin O.S., Zimonin D.V., Pavlenko N.I., Antonova A.B., Rubaylo A.I. Electrochemical study of new binuclear heterometallic vinylidene complexes with the Re-Pt bond. J. Sib. Fed. Univ. Chem. 2013. Vol. 1(6), P. 51-59.

13. Connelly N.G., Geiger W.E. Chemical redox agents for organometallic chemistry. Chem. Rev. 1996. Vol. 96(2), P. 877-910.

14. Antonova A.B., Chudin O.S., Pavlenko N.I., Sokolenko W.A., Rubaylo A.I., Vasiliev A.D., Verpekin V.V., Semeikin O.V. Chemistry of vinylidene complexes 19.* New heterometallic $\mu$-vinylidene complexes with Re-M (M = Pd, Pt) bonds. Molecular structure of $\left(\eta^{5}-\mathrm{C}_{5} \mathrm{H}_{5}\right)(\mathrm{CO}) \operatorname{RePt}(\mu-\mathrm{C}=\mathrm{CHPh})(\mu-$ CO) $\left(\mathrm{Ph}_{2} \mathrm{PCH}_{2} \mathrm{PPh}_{2}\right)$. Russ. Chem. Bull. 2009. Vol. 58(5), P. 955-963. 
15. Antonova A.B., Verpekin V.V., Chudin O.S., Vasiliev A.D., Pavlenko N.I., Sokolenko W.A., Rubaylo A.I., Semeikin O.V. Chemistry of vinylidene complexes. XXI. Synthesis, spectroscopic and structural study of the RePt and MnPt $\mu$-vinylidene complexes. Inorganica Chim. Acta. Elsevier B.V. 2013. Vol. 394, P. 328-336.

16. Johansson A.A., Antonova A.B., Pavlenko N.I., Rubaylo A.I. Infrared study of transformation of a terminal carbonyl ligand into a bridging one in the MnPt and MnPd $\mu$-vinylidene complexes. J. Mol. Structure. 1997. Vol. 408/409, P. 329-332.

17. Burmakina G.V., Verpekin V.V., Zimonin D.V., Chudin O.S., Rubaylo A.I. Effect of ligands coordinated at Platinum atom on redox properties of binuclear Manganese-Platinum phenylvinylidene complexes. J. Sib. Fed. Univ. Chem. 2018. Vol. 11(4), P. 543-551. 